\title{
La fundación del Hospital Infantil de México, ahora Hospital Infantil de México Federico Gómez
}

\author{
Manuel A. Baeza-Bacab ${ }^{1 *}$, Juan J. Luis Sienra-Monge ${ }^{2}$ y Horacio L. Reyes-Vázquez ${ }^{3}$ \\ ${ }^{1}$ Facultad de Medicina, Universidad Autónoma de Yucatán, Mérida, Yucatán; ${ }^{2}$ Hospital Infantil de México Federico Gómez, Ciudad de México; \\ ${ }^{3}$ Clínica Pigüi, Hospital Español, Ciudad de México. México
}

\section{Resumen}

En los primeros años del siglo XX, no existía en México un hospital que tuviera la capacidad de atender los problemas de salud de la niñez mexicana, lo que hacía necesaria la construcción de una institución moderna para atenderlos. En 1933, esta situación llevó a un grupo de médicos, encabezados por el Dr. Federico Gómez Santos, a solicitar y conseguir que el presidente de la República, Abelardo L. Rodríguez, reconociera la imperiosa necesidad de contar con un hospital de niños y aprobara el proyecto para su construcción. Luego de diez años de lucha en el campo político, social y económico, y con el apoyo de los presidentes Lázaro Cárdenas y Manuel Ávila Camacho, el 30 de abril de 1943 se inauguró el Hospital Infantil de México. Hoy, después de 75 años de su creación, el hospital ha resistido la prueba del tiempo y mantiene incólume sus principios de asistencia, enseñanza e investigación, emergiendo como la cuna de la pediatría mexicana y latinoamericana.

Palabras clave: Hospital. Pediatría. Historia. Infancia. México.

\section{The foundation of the Hospital Infantil de México, today Hospital Infantil de México Federico Gómez}

\begin{abstract}
In the early years of the $20^{\text {th }}$ century, no hospital in Mexico held the capacity to address the health problems of Mexican children, making it necessary to build a modern institution to take care of these issues. This situation mobilized a group of doctors led by Dr. Federico Gómez Santos to seek the acknowledgement of the President, Abelardo L. Rodríguez, of the urgent need of a children's hospital. Later, the President approved the project for its construction in 1933. After 10 years of struggle in the political, social and economic fields, and with the support of presidents Lázaro Cárdenas and Manuel Ávila Camacho, the Hospital Infantil de México was inaugurated on April $30^{\text {th }}, 1943$. Today, 75 years after its creation, the hospital has withstood the test of time maintaining intact its principles of assistance, teaching and research, and emerging as the cradle of Mexican and Latin American pediatrics.
\end{abstract}

Key words: Hospital. Pediatrics. History. Infancy. Mexico.

Disponible en internet: 13-05-2019 Bol Med Hosp Infant Mex. 2019;76:146-154 www.bmhim.com 


\section{Introducción}

Hace 75 años, el 30 de abril de 1943, luego de diez años de lucha en el campo político, social y económico, se inauguró el Hospital Infantil de México. Con el pasar del tiempo y en justo reconocimiento, adoptó el nombre de su fundador, Dr. Federico Gómez Santos, de quien el Dr. Jesús Kumate dijera «es el creador de la escuela pediátrica mexicana, fundador del Hospital Infantil y maestro de la pediatría latinoamericana" (Fig. 1) ${ }^{1}$. Por tanto, podemos concluir que su obra magna e inmortal, el Hospital Infantil de México, es la cuna de la pediatría nacional y latinoamericana.

El presente trabajo es tan solo una breve remembranza de su fundación y de los nombres de hombres y mujeres quienes, venciendo un cúmulo de obstáculos, hicieron posible esta invaluable obra que ha perdurado y se ha acrecentado con el tiempo. Es un modesto homenaje, no solo a aquellos que lo hicieron posible, sino a todos los individuos que a lo largo de 75 años han colaborado con su engrandecimiento.

\section{La atención infantil a principios del siglo $X X$}

En los primeros años del siglo XX, la atención de la salud infantil en la ciudad de México dependía de las salas de pediatría del Hospital General, del Hospital Juárez y, primordialmente, de la Casa de Niños Expósitos, mejor conocida como Casa Cuna. A partir de 1938, también empezó a funcionar un pequeño hospital para niños llamado Dolores Sanz de Lavie ${ }^{2}$.

En su trabajo Las tres primeras instituciones dedicadas a la asistencia hospitalaria pediátrica en México, el Dr. Jesús Lozoya Solís, uno de los fundadores del Hospital Infantil de México, señaló que el esplendor científico de la Casa Cuna se inició con la llegada del Dr. Manuel Cárdenas de la Vega a la dirección de dicha institución en 1931. Durante su administración, la Casa Cuna se trasladó a un nuevo local ubicado en la calle de Hidalgo, en Coyoacán (Fig. 2). La amplitud de las instalaciones permitió transformar un asilo de niños abandonados en un hospital donde se les atendía cuidadosamente desde un punto de vista integral. En este lugar se disponía de un servicio pediátrico como no había otro en México, ya que contaba con el personal necesario, médicos y enfermeras, y los servicios de otorrinolaringología, oftalmología, dermatología, laboratorio de análisis clínicos, gabinete de rayos $\mathrm{X}$, además de una cocina para la preparación de fórmulas especiales de alimentación ${ }^{3}$.

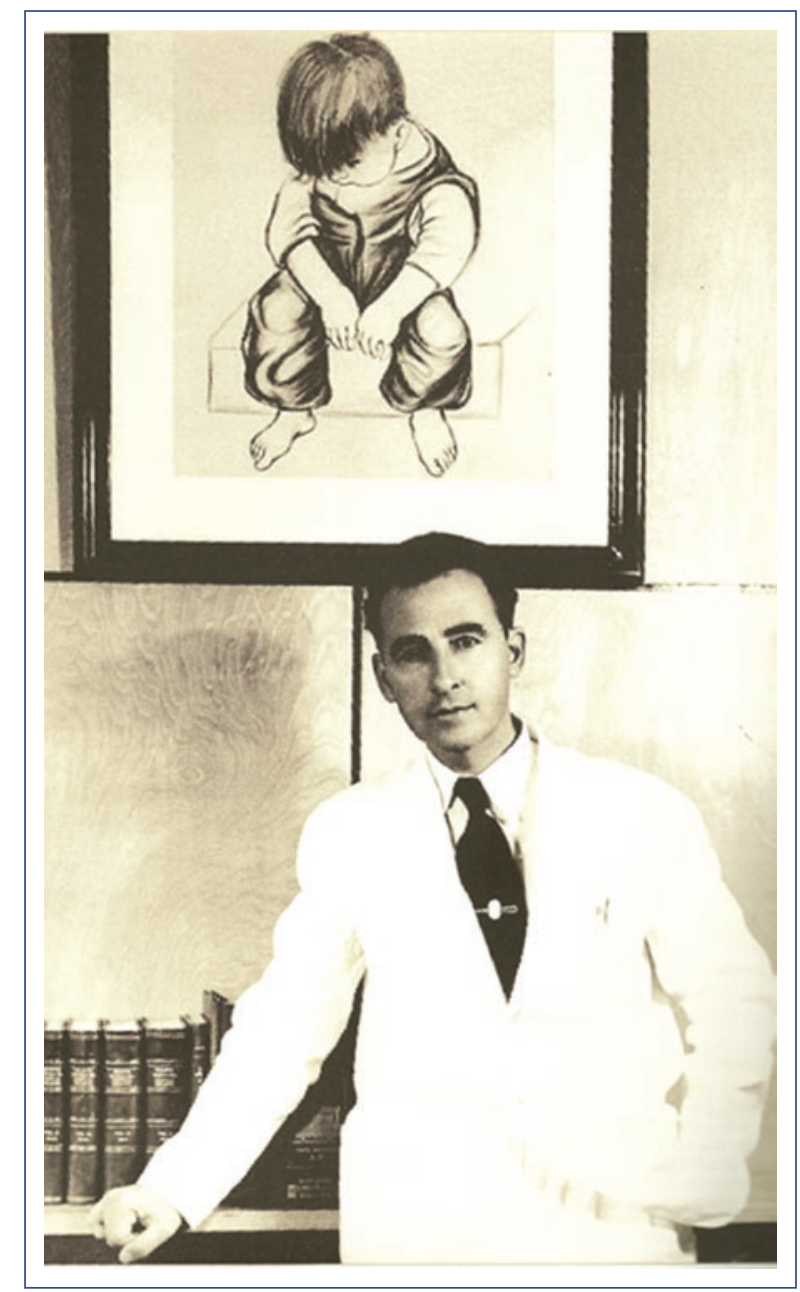

Figura 1. Dr. Federico Gómez Santos.

Se realizaba una historia clínica de todos los niños que ingresaban para elaborar un diagnóstico preciso de su estado de salud, e internarlos en las salas de niños sanos o de infecciosos, de acuerdo con su edad. En ese entonces, el responsable de la sala de lactantes era el Dr. Federico Gómez Santos, quien logró que la sala tuviera cubículos individuales con divisiones de madera y vidrio en lugar de cunas juntas en largas hileras ${ }^{3,4}$.

Lo anterior motivó que Rómulo Velazco escribiera lo siguiente: "La Casa Cuna, sin exagerar en lo mínimo, es el centro de perfeccionamiento de los médicos que se dedican a las enfermedades de los niños en la ciudad de México " 5 . Sin embargo, ni la Casa Cuna ni las salas de pediatría de los hospitales existentes tenían la capacidad para atender las necesidades de salud de la niñez mexicana, lo que hacía evidente la necesidad de un hospital para niños. 


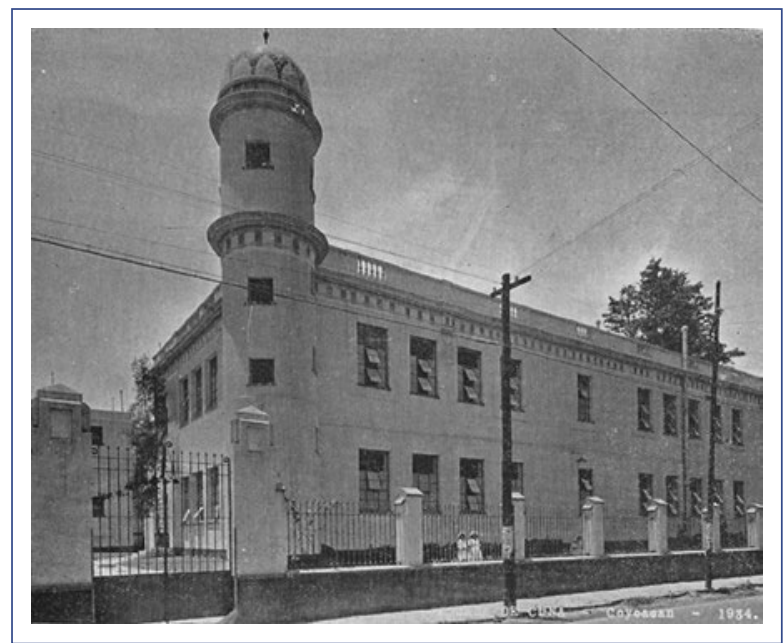

Figura 2. Casa Cuna, Coyoacán, 1934.

\section{Un entorno favorable}

El inicio de la tercera década del siglo XX fue afortunado para el desarrollo de la atención pediátrica nacional, ya que se realizaron varias acciones en favor de la infancia. Por ejemplo, los Congresos Mexicanos del Niño, patrocinados por el ingeniero Félix Palavicini, director del periódico El Universal durante 1921 y 1922 , los cuales reunieron a numerosos médicos, abogados y profesores para exponer su opinión sobre el estado en que vivían los niños mexicanos, así como las acciones que juzgaban precisas para reducir los altos índices de mortalidad, mejorar la alimentación, los hábitos de higiene y la educación, además de generar leyes adecuadas. Asimismo, en 1922, el Dr. Isidro Espinosa de los Reyes logró la construcción del primer Centro de Higiene Infantil; en 1927, con el apoyo del jefe del Departamento de Salubridad, Dr. Aquilino Villanueva, la creación del Servicio de Higiene Infantil, y dos años más tarde, la de la Asociación Nacional de Protección a la Infancia. Por otro lado, en 1938, el Dr. Espinosa reunió a un selecto grupo de médicos obstetras y pediatras en la Sociedad Mexicana de Puericultura. En 1940 se transformó en la Sociedad Mexicana de Pediatría, a la cual se afiliaron solamente los médicos que tenían una inclinación por la salud de los niños, particularmente los que trabajaban en los Centros de Higiene Infantil y en la Casa Cuna ${ }^{4,6}$.

La Sociedad sesionaba una vez al mes en el salón de actos de la Escuela Nacional de Medicina, donde se presentaban diversos trabajos orientados hacia los problemas pediátricos. Esto evidenció aún más la necesidad de servicios especializados para la atención de los niños. De tal manera, la Sociedad se convirtió en un semillero de entusiasmo, de proyectos y de ideas para el avance de la nueva tendencia pediátrica en México. Al término de las reuniones científicas, los asistentes rutinariamente terminaban en el Café de Tacuba, donde vertían, durante horas, animados comentarios y sugerencias para contar con un hospital de niños en México ${ }^{4}$.

A decir del Dr. Lozoya, otro sitio importante de reuniones fue el domicilio del Dr. Mario A. Torroella, subjefe de Servicio de Higiene Infantil, donde organizaba veladas científico-literarias, con la participación de Manuel Cárdenas de la Vega, Federico Gómez, Rigoberto Aguilar Pico, Pablo Mendizábal y el joven arquitecto José Villagrán García, fincando con gran perspicacia las bases de un trato amistoso y fecundo, que se convertiría en una de las características de los pediatras mexicanos. A decir del autor, de esas reuniones surgió el primer proyecto para la construcción y organización del Hospital Infantil de México ${ }^{3}$.

Sin embargo, el medio político no parecía darse cuenta del desamparo de los niños enfermos ni de la creciente inquietud pediátrica. Así las cosas, el Dr. Federico Gómez recuerda que su práctica privada lo llevó a atender a los nietos de don Pedro Malabehar Peña, jefe de editorialistas del periódico El Universal, quien se interesó en la idea de construir en México un moderno hospital para niños, por lo que puso a disposición del Dr. Gómez las columnas de su página editorial. A partir de ese momento, una vez por semana durante cuatro o cinco años, aparecieron artículos dirigidos al gobierno y al público en general, que describían lo que varios países hacían en favor de sus niños para protegerlos en la salud y la enfermedad. Todos los artículos terminaban con un mensaje angustioso, que enfatizaba la necesidad de fundar una institución moderna que se hiciera cargo del gran número de niños enfermos ${ }^{4}$.

\section{La primera propuesta}

Aunque el gobierno federal, en 1920, y la Cruz Blanca Neutral, en 1922, tuvieron la intención de crear un hospital para niños, los proyectos nunca progresaron. La oportunidad formal se presentó con los cambios políticos de 1932, que llevaron al General Abelardo L. Rodríguez, prominente militar y político del noroeste, a la Presidencia de la República. Su médico personal, el Dr. Mariano Romero Ochoa, era amigo de los doctores Rigoberto Aguilar Pico y Federico Gómez, y los había escuchado en diversas ocasiones hablar con entusiasmo y convicción acerca de la apremiante necesidad de un hospital para niños. El Dr. Romero, simpatizante de esa idea a 
pesar de no ser pediatra, se comprometió a entregar al presidente Rodríguez una carta firmada por los tres, en la cual se solicitaba que el gobierno diera los pasos necesarios para que la niñez mexicana contara con un hospital. Después de varias semanas de impaciencia y de dudas, el presidente de la República envió una carta de respuesta, fechada el 31 de mayo de 1933: «Encuentro que, efectivamente, existe la imperiosa necesidad de contar con un hospital para niños, y con todo gusto trataré el asunto con el jefe del Departamento Central, así como con el director de la Beneficencia, con el objeto de estudiar y llevar a cabo su proyecto» ${ }^{4,7}$.

Poco después, se reunieron el jefe del Departamento Central, Lic. Aarón Sáenz, y el director de la Beneficencia Pública, Gral. José María Tapia, y decidieron que un grupo de ingenieros se hiciera cargo del proyecto y construyera el tantas veces mencionado hospital. Por fin, se nombró una comisión que asesorara a los ingenieros constructores. Esta comisión estaba formada por el Dr. Fernando Ocaranza, vocal médico de la Junta Directiva de la Beneficencia; Dr. José Castro Villagrana, director del Hospital Juárez; Dr. Manuel Guevara Oropeza, director del manicomio La Castañeda; y Dr. Manuel Cárdenas de la Vega, director de la Casa Cuna ${ }^{4}$.

Al conocerse el inicio de las obras del Hospital del Niño, el Dr. Federico Gómez refiere que la Sociedad Mexicana de Puericultura convocó a una movida sesión, en la que se decidió ofrecer a la comisión de médicos de la Beneficencia Pública la colaboración de un grupo de pediatras aptos para asesorar el proyecto en sus aspectos funcionales y en la distribución general y capacidad de los distintos servicios. Dicha comisión quedó formada por el Dr. Mario Torroella, el Dr. Pablo Mendizábal y el propio Dr. Federico Gómez. Los involucrados se presentaron con gran entusiasmo ante el Dr. Fernando Ocaranza, vocal médico de la Beneficencia Pública, quien acabó con las ilusiones del grupo al emitir un rotundo rechazo pues, según el Dr. Ocaranza, la comisión formada por el Departamento Médico de la Beneficencia Pública era suficientemente capaz para asesorar al Departamento de Ingenieros para la construcción del Hospital del Niño, por lo que dio oficialmente las gracias a la Sociedad Mexicana de Puericultura por su ofrecimiento y despachó la comisión con cajas destempladas ${ }^{4}$.

\section{Inician los trabajos de planeación y construcción}

A partir de 1933 dieron inicio los trabajos de planeación del nuevo hospital. Las ideas más claras provinieron del Dr. Manuel Cárdenas de la Vega quien señaló la necesidad de discriminar los niños que requerían hospitalización, la posibilidad de internar a la madre junto con el niño, brindar una atención especializada basada en la disponibilidad de médicos y enfermeras especialistas, separar a los niños de acuerdo con su edad y contar con un pabellón de pensionistas para dar respuesta a la demanda de atención hospitalaria de personas con recursos económicos suficientes. Después de dos meses de trabajos, se logró arribar a la idea de construir un edificio de varios pisos con 500 camas de acuerdo con las necesidades calculadas 8 .

Para diciembre de 1933, ya se contaba con los planos del hospital elaborados por el ingeniero Alberto Barocio, eligiendo una construcción con planta en forma de peine con seis prolongaciones, sobre las que se planeaban solarios y terrazas. El terreno elegido para la construcción del Hospital del Niño estaba localizado detrás del Hospital General: un terreno de $20,000 \mathrm{~m}^{2}$ donde se sembraban hortalizas. La construcción se inició el 3 de marzo de 1934.

\section{Las primeras nubes de tormenta}

La primera mala noticia para el naciente proyecto fue la muerte prematura, el 26 de octubre de 1934, del Dr. Manuel Cárdenas de la Vega. El acontecimiento pareció detener los procesos de superación de la Casa Cuna y la construcción del hospital. Sin embargo, ambos proyectos cayeron bajo la dirección del Dr. Federico Gómez, quien fue comisionado asesor médico del Departamento de Ingeniería de la Beneficencia Pública para la construcción del Hospital del Niño2,4.

Para finales de 1934, la obra había avanzado vertiginosamente, aunque el Dr. Gómez decía que la construcción exhibía gran número de errores funcionales, producto del desconocimiento de lo que debía ser un hospital para niños. Cuando se discutían esos problemas, los remates del gran peine en el sector oriente y poniente comenzaron a hundirse, lo que causó gran preocupación a los constructores y las autoridades 4 .

El Dr. Eugenio Toussaint Aragón, otro de los fundadores del hospital, describió esa experiencia de la siguiente manera: «En los terrenos del Hospital General, en la colonia de los Doctores, lentamente se fue elevando una estructura metálica sobre un piso de consistencia lodosa, lo que poco antes eran lotes baldíos que se anegaban en tiempos de lluvias formando lagunas, donde los llamados perros de agua, pequeños patos, se detenían en su vuelo hacia el Lago de Texcoco. Diariamente, pasaba a la orilla de esta zona a 
bordo del tranvía eléctrico, cruzando a través de terrenos de siembra, que luego serían las avenidas Baja California y Calzada de la Piedad (hoy, Avenida Cuauhtémoc). Por esa circunstancia, pude contemplar cómo el esqueleto de acero del próximo edificio iba ganando altura, hasta llegar al quinto nivel, donde se detuvo: la estructura comenzó a hundirse y desnivelarse, ya que las lagunas habían sido rellenadas con basura de la colonia de los Doctores y no tenían la firmeza para tolerar el peso de una obra de gran envergadura» ${ }^{6}$.

Aunado a lo anterior, en la década de 1930, la experiencia en México sobre mecánica de suelos era muy pobre, lo que afectó seriamente la cimentación del hospital. Con gran decepción, la obra tuvo que suspenderse para estudiarla, y a pesar de aplicar medidas para cimentar nuevamente, nunca dieron el menor beneficio no obstante su elevado costo. Por casi tres años, el tan ardientemente esperado hospital fue abandonado a su suerte. La decepción cundió en la naciente pediatría mexicana, donde solo se escuchaban los tristes comentarios de una situación que se preveía irremediable 4 .

Los movimientos políticos en México trajeron cambios en las secretarías de estado. Con cada cambio se iniciaba una nueva peregrinación por antesalas y pasillos, tratando de convencer a las nuevas autoridades para que pusieran remedio a la agrietada construcción del hospital y a la herrumbre de las trabes de acero que comenzaban a carcomerse. Sin embargo, no se logró despertar interés por el problema en ninguna autoridad. Para entonces, desapareció la Beneficencia Pública y se creó la Secretaría de Asistencia para sustituirla ${ }^{4}$.

\section{Una nueva esperanza}

A finales de 1934 llegó a la Presidencia de la República el Gral. Lázaro Cárdenas, quien designó como encargado de la Secretaría de Asistencia al Lic. Silvestre Guerrero. En ese entonces, el Dr. Federico Gómez fue llamado como pediatra a la residencia de Los Pinos, para atender al hijo recién nacido del presidente. Cada dos semanas visitaba a su paciente, siempre esperando una oportunidad para hablar del Hospital del Niño, aún en construcción. Con la asesoría de la secretaria de la residencia presidencial (la señorita Vázquez Gómez), elaboró un proyecto para agrupar a todas las oficinas dispersas relacionadas, en una o en otra forma, con la protección del niño sano y la atención del niño enfermo, con lo cual podría absorberse el problema del Hospital del Niño, cuya construcción estaba abandonada ${ }^{4,6}$.

La propuesta del Dr. Gómez fue aprobada y el Gral. Cárdenas creó el Departamento Autónomo de Asistencia Social Infantil, nombrando como director a su médico personal, el Dr. Salvador Zubirán, y como secretario general, al Dr. Federico Gómez. Los primeros acuerdos de dicho departamento fueron para realizar un estudio que pusiera a flote el Hospital del Niño, cuya obra estaba suspendida desde hacía varios años. Estudios fueron y vinieron sin llegar a ninguna conclusión viable, hasta que se llamó al destacado arquitecto José Villagrán García para asesorar el proyecto. Sin embargo, vinieron cambios al gabinete del Gral. Cárdenas, y el flamante Departamento fue incorporado a la Secretaría de Asistencia Social ${ }^{4}$.

Ante la presión social y médica para seguir la construcción, el presidente convocó a una junta de ingenieros y arquitectos autores de varios estudios. A decir del Dr. Gómez, el presidente los escuchó con la adusta paciencia de su carácter, y al final pronunció la pregunta clave: "¿Esa gran estructura de acero remachada como una gran jaula podría derrumbarse, poniendo en serio peligro al personal y a los enfermos por el hecho de estar desnivelada su estructura en los extremos?" Los ingenieros y arquitectos contestaron: «El armazón de acero unido a la estructura no puede fraccionarse a pesar de los hundimientos parciales de sus extremos. Los movimientos en conjunto implican un peligro mínimo para el personal y los enfermos». "En este caso - repuso el presidente-, dispongo que se continúe la obra, se equipe y se ponga en funciones. La Secretaría de Asistencia proveerá el presupuesto para ese fin». Por tal motivo, el 20 de junio de 1938 quedó autorizada la conclusión del hospital, formándose una comisión integrada por el arquitecto José Villagrán García y los doctores Federico Gómez Santos, Jorge Muñoz Turnbull y Roberto Sánchez Messieck ${ }^{4,8}$.

Después de una larga fase de indecisiones, la obra se continuó y los pediatras volvieron a tener la esperanza de encontrar un albergue para los niños enfermos y un estímulo apropiado para sus inquietudes de servicio y enseñanza.

\section{El final añorado}

La actividad en la obra del Hospital del Niño, impulsada por José Villagrán García, era una efervescencia de trabajo, ideas y proyectos. Al iniciar la década de 1940, se avizoraron cristalizaciones. El nuevo presidente de la República, Gral. Manuel Ávila Camacho, 
designó, para fortuna de la pediatría mexicana y en particular del hospital en construcción, como secretario de Asistencia Pública al Dr. Gustavo Baz Prada y como subsecretario al Dr. Salvador Zubirán $n^{4,6}$.

En ese entonces fue necesario salvar un nuevo obstáculo: la Segunda Guerra Mundial, ya que los materiales y el equipo necesarios se adquirían en el extranjero y habían empezado a escasear. Sin embargo, se obtuvo presupuesto para enviar a los médicos que iban a ser jefes de servicio a hospitales en los Estados Unidos, a fin de que conocieran programas de trabajo y de organización técnica y administrativa. De conformidad con Viesca y Díaz, para 1942 podía afirmarse que la Secretaría de Asistencia ya había becado a diez médicos en el extranjero. Algunos de ellos se encontraban ya de regreso, y los campos de especialización de su elección eran la Medicina, Cirugía, Anestesiología, Fisioterapia, Anatomía Patológica y Técnicas Hospitalarias. Desafortunadamente, los informes existentes no consignan los nombres de los médicos becados. Sin embargo, todo parece indicar que entre ellos estaban Jesús Lozoya Solís, Alejandro Aguirre, Carlos Herrera, Felipe Cacho y Guillermo de Ovando ${ }^{8}$. Otro médico que aparentemente también fue becado para prepararse fue el Dr. Rigoberto Aguilar Pico, pues el mismo Dr. Aguilar señala: «Aún recordamos la extensa actividad previa a la inauguración desarrollada por el Dr. Gómez y sus colaboradores. Recordamos también el entusiasmo con que todos los designados como jefes de servicio salimos al extranjero, principalmente a los Estados Unidos, para visitar y estudiar en los hospitales pediátricos más acreditados" "9. Asimismo, se llevó a cabo un curso de enfermería pediátrica para enfermeras tituladas, eligiendo entre las que aprobaron el curso a un pequeño grupo que fue enviado para perfeccionar sus conocimientos al Hospital de Niños y la Escuela de Enfermería de la Universidad North Western de Cleveland, Ohio, bajo la dirección de la que sería la jefa de enfermeras del hospital, Carmen Gómez Siegler (Fig. 3) $)^{4,8}$.

Al regresar de su aprendizaje, enfermeras y médicos se dedicaron a enseñar en sus respectivos campos lo que habían aprendido, desarrollando cursos de adiestramiento para todo el personal que pretendía ingresar al hospital, lo que obligó a poner en funciones, en primer lugar, las aulas, para ofrecer los cursos mencionados $4,6,10$.

Cabe mencionar que, en su informe de septiembre de 1942, el presidente Ávila Camacho mencionó por primera vez la institución, en forma oficial, como Hospital Infantili1.

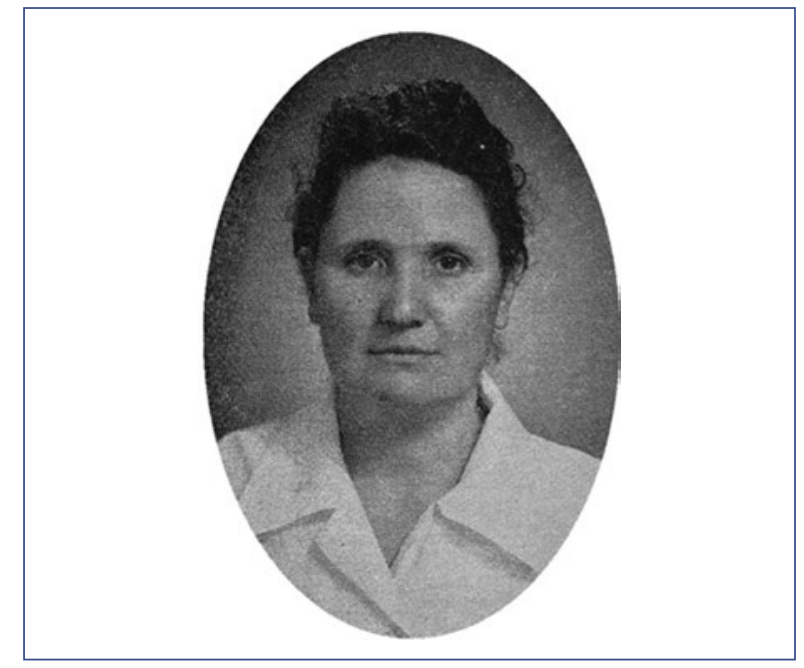

Figura 3. Enfermera Carmen Gómez Siegler, primera jefa de enfermeras del Hospital Infantil de México, 1943.

\section{La inauguración}

A principios de 1943, se estimaba que la capacidad del hospital sería de 600 camas distribuidas en el cuerpo del edificio, cuya disposición se mantuvo como un cuerpo central con seis prolongaciones a manera de «dientes de peine». Los servicios se distribuyeron de la siguiente manera: en el subsuelo, fisioterapia, farmacia central, sección para madres asiladas, admisión y depósito de ropa de niños asilados, cocina central, comedor del personal, almacenes generales, mortuorio y anfiteatro de cadáveres; en el primer piso se ubicarían el servicio de rayos $\mathrm{X}$, trabajo social, policlínica para externos, urgencias, banco de sangre, cirugía dental, oficinas de gobierno y vestidores para médicos y visitantes, laboratorios (incluyendo el de investigación con su bioterio, anatomopatológico y experimental), servicio de fotografía y microfotografía, cocina, esterilización y toma de productos; el segundo piso albergaría las salas de medicina para niños de 2 a 14 años y la sección de enseñanza con su aula, biblioteca y archivos; el tercero se destinaría a servicios mixtos, prematuros, lactantes, especialidades médicas y quirúrgicas, como enfermedades de la piel y sífilis, oftalmología y otorrinolaringología, laboratorio de leches y la oficina y habitaciones de los médicos internos; el cuarto sería para los servicios quirúrgicos; y, finalmente, el quinto se dedicaría a los pacientes tuberculosos e infecciosos y las habitaciones y el aula para las médicas internas ${ }^{8}$. 
El 17 de abril de 1943, los periódicos de la época anunciaron la inauguración del hospital para el último día de ese mes. En una de las notas, además de leer el papel que el ya casi inaugurado hospital debería jugar en la enseñanza, el Dr. Federico Gómez hizo una declaración trascendental al afirmar que dejaría su práctica privada para dedicarse exclusivamente al hospital, situación que ya venía realizando, pues había habilitado una oficina en lo que sería el sótano del hospital ${ }^{10}$. En ese entonces, ya se había fijado el número y naturaleza del personal que trabajaría en el Hospital Infantil, constituido por el personal que participaría parcialmente en el trabajo hospitalario y los que vivirían en el hospital, entre los que se encontraban el Dr. Federico Gómez Santos como director; cuatro residentes (Jesús Lozoya Solís y Felipe Cacho, residente y ayudante de cirugía; Alejandro Aguirre y Carlos Herrera, residentes de medicina, y Fernando Madero y Rodolfo Muñoz Enciso como sus ayudantes); 18 médicos internos; el administrador, y la jefa de enfermeras, Carmen Gómez Siegler ${ }^{8}$.

La primera generación de médicos internos estuvo formada por cuatro mujeres y 14 hombres: Raúl Ávila, Lázaro Benavides, Beatriz Bienvenu, Francisco Cisneros, Fernando Espinosa Ogarrio, Marcelino Figueroa, Guillermina García, Alfonso González Ordóñez, Carmen Labra Robredo, Antonia E. Machain, Manuel Méndez Matías, José Martínez Posada, Heriberto Montemayor, Rodolfo Quintero, Elías Romero Sánchez, Carlos Vidales, Rubén Lavalle y Guillermo Zárate Mijangos $^{6,8}$. Los médicos externos fueron Carlos Abad Martínez, Rigoberto Aguilar Pico, Jesús Álvarez de los Cobos, Luis Berlanga Berumen, Manuel Berumen, Hermilo Castañeda, Gustavo Castillo Díaz, Nicandro Chávez, Guillermo De Ovando A., Juan Farril, Demófilo González, Daniel Gurría Urgell, Luis Gutiérrez Villegas, Fernando López Clares, Artemio Gallegos Landeros, Rafael Moreno Valle, Jorge Muñoz Turnbull, Dionisio Pérez Cosío, Antonio Ramírez Ciará, Fermín Reygadas Macedo, Rafael Ramos Galván, Vicente Roqueñí, Maximiliano Salas Martínez, Roberto Sánchez Messieck, Rafael Soto Allande, Herbert Stacpoole, Antonio Torres de Anda, Eugenio Toussaint Aragón, Luis Vargas y Vargas y Alejandro Velasco Zimbrón ${ }^{6,8}$.

Días antes de la fecha prevista, el secretario de Asistencia Pública, Dr. Gustavo Baz, giró las invitaciones a la ceremonia de inauguración del Hospital Infantil que tendría lugar el 30 de abril de 1943, contando con la presencia del C. Presidente de la República. En esta invitación oficial se denomina a la institución Hospital Infantil, la cual vendría a substituir al de Hospital del

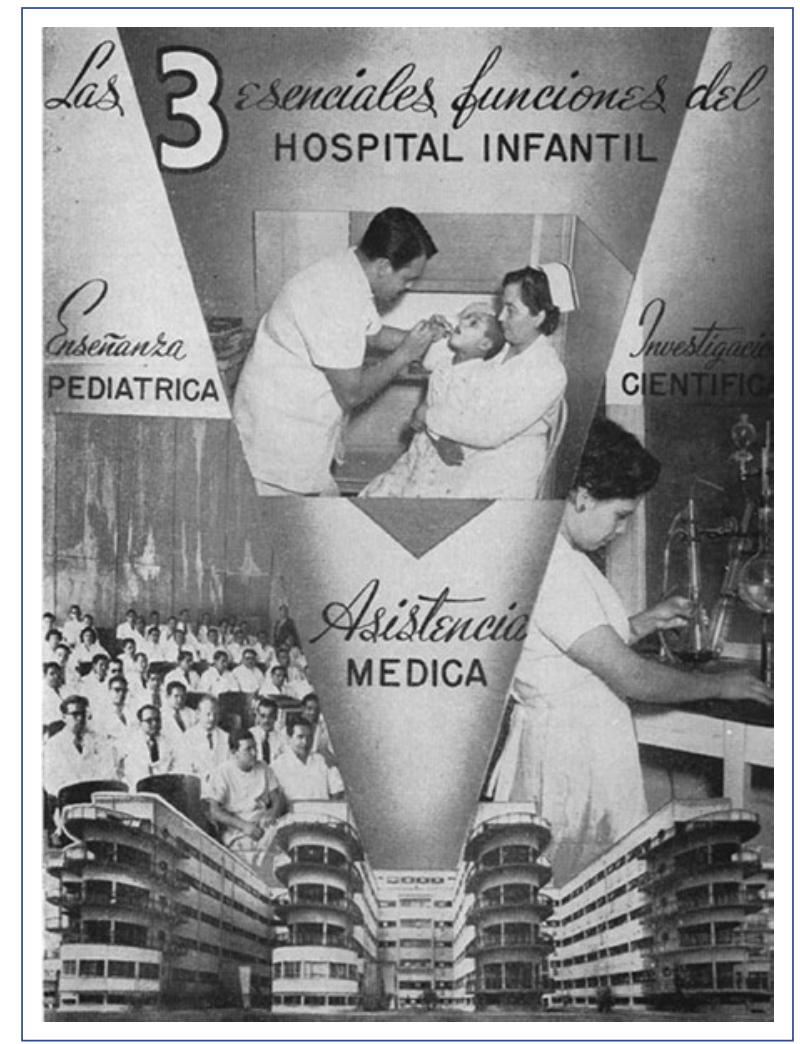

Figura 4. Las tres funciones esenciales del Hospital Infantil de México Federico Gómez.

Niño, como se conoció desde su inicio. El Dr. Toussaint señala que los puristas del lenguaje habían criticado el nombre de «infantil», porque hacía alusión a pequeño. Sin embargo, el público aceptó el nombre sin tomar en cuenta a la Academia de la Lengua ${ }^{6}$, y quedó ratificada su denominación con la promulgación de la ley de su creación publicada en el Diario Oficial el 23 de junio de 1943, la cual dice lo siguiente:

Artículo $1^{\circ}$. Se crea el Hospital Infantil en la ciudad de México, con el objeto de llenar tres funciones esenciales:

a) Dar atención médica, en todos sus aspectos, a los niños enfermos, fundamentalmente a los niños indigentes y débiles económicos.

b) Propagar, por todos los medios a su alcance, la enseñanza de la Pediatría a médicos y estudiantes de Medicina y la enseñanza de la Enfermería Pediátrica a enfermeras graduadas.

c) Fomentar la producción científica y la investigación de los problemas médico-sociales de la niñez mexicana $^{12}$ (Fig. 4).

A pesar de los anuncios previos, el presidente de la República no asistió a la inauguración, al parecer por 
una infección respiratoria, pero estuvo representado por su hermano, el Gral. Maximino Ávila Camacho, secretario de Comunicaciones y Obras Públicas, a quien acompañaron el Lic. Miguel Alemán Valdés, secretario de Gobernación, el Dr. Gustavo Baz, secretario de Asistencia Pública, y el Gral. Lázaro Cárdenas, secretario de la Defensa Nacional, entre otros ${ }^{6,10}$.

En su discurso inaugural, el Dr. Federico Gómez señaló lo siguiente: «Estamos celebrando hoy la ansiada inauguración del Hospital y ya tenemos enfrente la idea realizada. Ahora, nos toca a los médicos y al personal que vamos a colaborar en la institución hacerla marchar, crecer y prestigiarse, dando a los niños enfermos de México el servicio más completo y eficiente que pueda darse a la infancia en cualquier país de la tierra. La idea es ambiciosa, pero es realizable y la llevaremos a cabo. Nuestra meta será hacer de este hospital una institución que llene tres importantes funciones: un excelente servicio a la sociedad, un propicio campo para la enseñanza pediátrica y un ambiente fecundo para la investigación de los problemas patológicos de la infancia. La función social dará un refugio acogedor al niño enfermo, procurando que el cariño y la simpatía le hagan menos amarga su estancia en el hospital. La función de enseñanza abrirá sus puertas a los médicos, a los estudiantes y a las enfermeras de la capital y de los estados que deseen especializarse y después volver a su tierra natal a propagar conocimientos que defiendan la salud y la vida de los niños. La función de investigación tenderá a esclarecer muchos hondos problemas de las enfermedades de los niños de México, y sus resultados en forma de producción científica irán a todos los rincones de la República, repartiendo entre los médicos fruto abundante y óptimo»?

Diez años después, en su discurso de aniversario, el fundador dijo lo siguiente: «Si el Hospital Infantil resiste la prueba de los años sin caer en la rutina inútil y somnolienta, alentada por el permanente espíritu de inquietud que ahora lo mueve, no se tambaleará porque cambien sus dirigentes, ni sentirá amenaza ni zozobra porque las autoridades se renueven, ni temblará espantado ante las embestidas de los incomprensivos. Simplemente seguirá su camino, mirando con serenidad la agitación pasajera, fijando la vista en sus tres postulados, llenos de anhelos, de responsabilidades y de exquisitas satisfacciones: servicio médico eficiente a la niñez enferma, difusión ilimitada de la enseñanza pediátrica e investigación ${ }^{13}$.

Hoy, a 75 años de su creación, se puede decir al fundador: ¡Hemos cumplido!

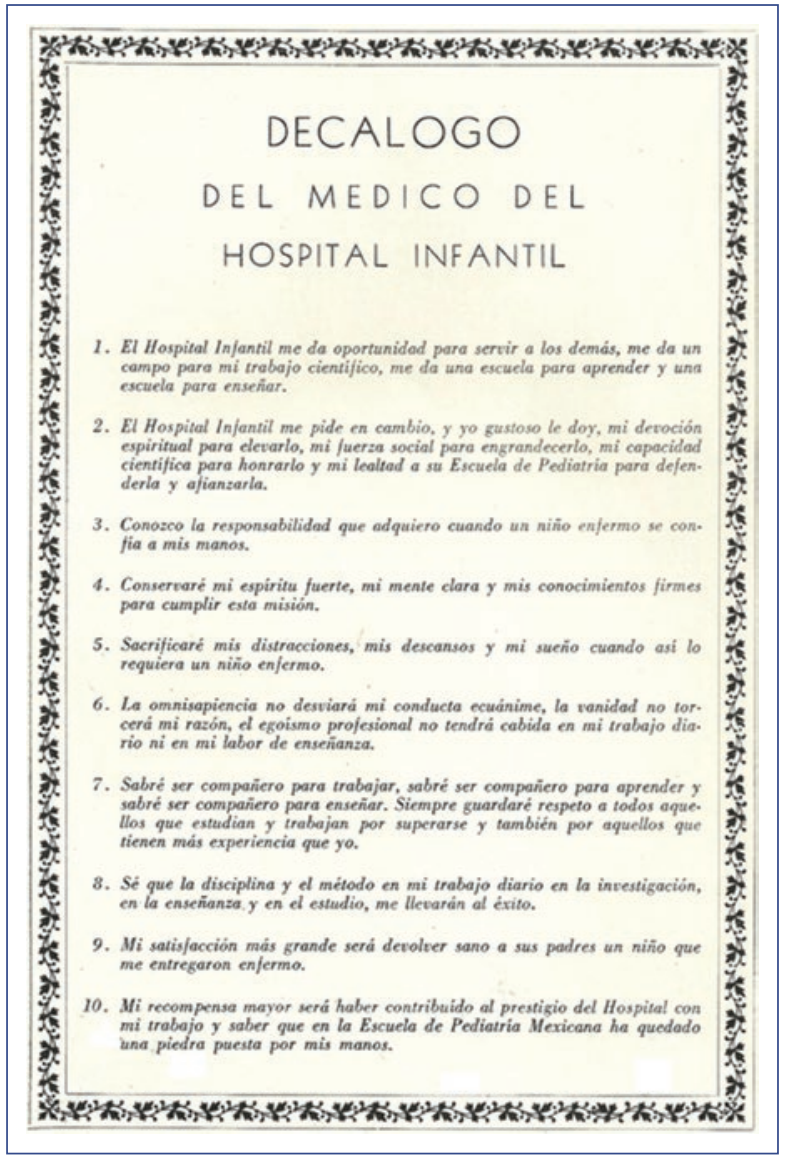

Figura 5. Decálogo del Médico del Hospital Infantil de México.

El Hospital Infantil de México Federico Gómez ha resistido la prueba del tiempo y mantiene incólume sus principios de asistencia, enseñanza e investigación, cumpliendo con el compromiso que estableció el fundador en el inciso diez del Decálogo del Médico del Hospital Infantil de México: «Mi recompensa mayor será haber contribuido al prestigio del Hospital con mi trabajo y saber que en la Escuela de Pediatría Mexicana ha quedado una piedra puesta por mis manos" (Fig. 5).

\section{Conflicto de intereses}

Los autores declaran no tener ningún conflicto de intereses.

\section{Bibliografía}

1. Kumate J. Federico Gómez Santos (1897-1980). Bol Med Hosp Infant Mex. 1996;53:300-2.

2. Viesca-Treviño C. VI. Los orígenes de la pediatría. Gac Med Mex. 1994;130:510-6. 
3. Lozoya-Solís J. Las tres primeras instituciones dedicadas a la asistencia hospitalaria pediátrica en México. Prensa Med Mex. 1966;104-29.

4. Gómez F. La atención al niño enfermo a partir de la Independencia. En: Ávila-Cisneros I, Padrón-Puyou F, Frenk S, Rodríguez-Pinto M, editores. Historia de la pediatría en México. México: Fondo de Cultura Económica; 1997. p. 311-32.

5. Velasco Ceballos R. El niño mexicano ante la caridad y el estado. México: Editorial Cultura; 1935.

6. Toussaint-Aragón E. Hospital Infantil de México «Dr. Federico Gómez» 1943-1983. México: Imprenta Aldina; 1983.

7. Gómez F, Benavides L. Hospital Infantil México, D.F. 1943-1953. Informe de Labores. México: Talleres Gráficos de la Nación; 1953.

8. Viesca-Treviño C, Díaz-De Kuri M. Hospital Infantil de México Federico
Gómez, medio siglo de historia. México: Gráfica, Creatividad y Diseño, S.A de C. V; 2001.

9. Aguilar-Pico R. El Hospital Infantil de México. Gac Med Mex. 1963;93: 1155-64.

10. Chico-Ponce de León F. Crónica hemerográfica de la fundación del Hospital Infantil de México Federico Gómez. Bol Med Hosp Infant Mex. 2010;67:1-49.

11. Cámara de Diputados LX Legislatura. II Informe de Gobierno del Presidente Constitucional de los Estados Unidos Mexicanos Manuel Ávila Camacho. $1^{\circ}$ de septiembre de 1942. México; 2006.

12. Diario Oficial de la Federación de México (1943, 23 de junio). Ley que crea el Hospital Infantil en esta ciudad.

13. Gómez Santos F. Palabras del director (inédito). Bol Med Hosp Infant Mex. 1968;25:48-9. 